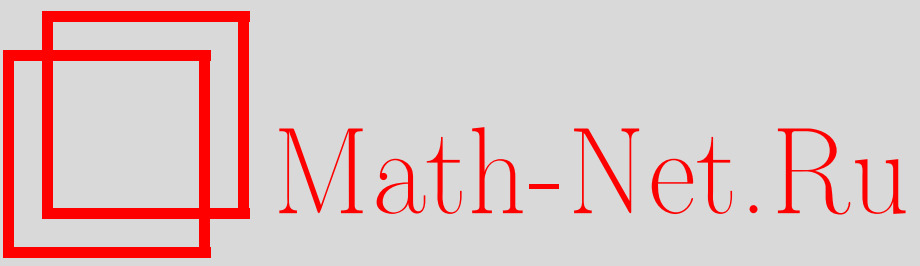

И. В. Баскаков, Тройные произведения Масси в когомологиях момент-угол комплексов, УМН, 2003, том 58, выпуск 5, 199-200

DOI: https://doi.org/10.4213/rm670

Использование Общероссийского математического портала Math-Net.Ru подразумевает, что вы прочитали и согласны с пользовательским соглашением

http://www . mathnet.ru/rus/agreement

Параметры загрузки:

IP : 3.81 .55 .215

26 апреля 2023 г., 14:32:43 


\title{
ТРОЙНЫЕ ПРОИЗВЕДЕНИЯ МАССИ В КОГОМОЛОГИЯХ МОМЕНТ-УГОЛ КОМПЛЕКСОВ
}

\author{
И.В. БАСКАКОВ
}

Основная цель настоящей работы - показать, что комбинаторное определение умножения в когомологиях момент-угол комплексов [1] позволяет строить большой класс момент-угол комплексов с нетривиальным произведением Масси в их когомологиях.

ОПреДЕлЕнИЕ. Пусть $K$ - симплициальный комплекс, $V=\left\{v_{1}, \ldots, v_{m}\right\}$ - множество его вершин, момент-угол комплексом $K$ назьвается подпространство полидиска

$$
\mathscr{Z}_{K}=\bigcup_{\sigma \in K}\left(D^{2}\right)^{\sigma} \times\left(\partial D^{2}\right)^{V \backslash \sigma} \subset\left(D^{2}\right)^{V},
$$

где $D^{2} \subset \mathbb{C}$ - стандартньй диск.

Более подробно все необходимые ниже факты о момент-угол комплексах содержатся в [2], где построено клеточное разбиение момент-угол комплекса и описана мултиградуированная алгебра его клеточных коцепей. Итак, следуя [2], рассмотрим клеточное разбиение полидиска $\left(D^{2}\right)^{V}$, индуцированное стандартным трехклеточньм разбиением $D^{2}$, и заметим, что для любого $K$ момент-угол комплекс $\mathscr{Z}_{K}$ является клеточньп подпространством полидиска. В [2] также показано, что клеточные коцепи $\left(D^{2}\right)^{V}$ можно снабдить структурой $\mathbb{Z}^{V} \oplus \mathbb{Z}$-градуированной алгебры, изоморфной $\mathbb{k}\left[v_{1}, \ldots, v_{m}\right] \otimes \Lambda\left[u_{1}, \ldots, u_{m}\right] /\left(v_{i}^{2}=0, v_{i} u_{i}=0\right)$, где $\operatorname{mdeg} v_{i}=(0, \ldots, 0,2,0, \ldots, 0 ; 0), \operatorname{a~mdeg} u_{i}=(0, \ldots, 0,2,0, \ldots, 0 ;-1)$, двойки стоят на $i$-х местах. Дифференциал задается равенствами $d=0, d v_{i}=0$ и $d u_{i}=v_{i}$. Клеточныекоцепи $\mathscr{Z}_{K}$ являются фактор-алгеброй клеточных коцепей полидиска по идеалу, порожденному мономами вида $v_{i_{1}} \cdots v_{i_{l}}$, где $\left\{v_{i_{1}}, \ldots, v_{i_{l}}\right\} \notin K$.

Необходимо отметить, что дифференциал сохраняет $\mathbb{Z}^{V}$-градуировку, и таким образом комплекс клеточных коцепей распадается в прямую сумму подкомплексов, каждый из которых изоморфен комплексу пополненных симплициальных коцепей некоторого полного подкомплекса $K$. Это позволяет доказать следующую теорему, которая понадобится нам для вычисления тройного произведения Масси.

Tеорема ([1]). Имеет место изоморфизм: $H^{*}\left(\mathscr{Z}_{K}\right)=\bigoplus_{\gamma \subset V} \widetilde{H}^{*-|\gamma|-1}\left(K_{\gamma}\right)$, где $K_{\gamma}=\{\sigma \in K \mid \sigma \subset \gamma\}$ - полный подкомплекс $K$ на вершинах из $\gamma$. Кроме того, если $a_{1} \in \widetilde{H}^{*}\left(K_{\gamma_{1}}\right)$ u $a_{2} \in \widetilde{H}^{*}\left(K_{\gamma_{2}}\right)$, mo:

$$
a_{1} a_{2}= \begin{cases}0, & \gamma_{1} \cap \gamma_{2} \neq \varnothing \\ i_{\gamma_{1}, \gamma_{2}}^{*} \lambda^{*}\left(a_{1} \otimes a_{2}\right), & \gamma_{1} \cap \gamma_{2}=\varnothing\end{cases}
$$

əде $\lambda: \widetilde{C}^{*}\left(K_{\gamma_{1}}\right) \otimes \widetilde{C}^{*}\left(K_{\gamma_{2}}\right) \rightarrow \widetilde{C}^{*+1}\left(K_{\gamma_{1}} * K_{\gamma_{2}}\right)-$ изоморфизм, $i_{\gamma_{1}, \gamma_{2}}: K_{\gamma_{1}} \sqcup \gamma_{2} \rightarrow K_{\gamma_{1}} * K_{\gamma_{2}}$ - вложение, а $K_{\gamma_{1}} * K_{\gamma_{2}}$ - джойн $K_{\gamma_{1}}$ u $K_{\gamma_{2}}$.

Теперь напомним определение тройного произведения Масси.

Пусть $\left[C^{*}, d\right]$ - некоторая дифференциальо-градуированная алгебра и $a \in C^{p}$. Положим $\bar{a}=(-1)^{p} a$

ОПредЕлЕниЕ. Пусть $a, b, c \in C^{*}$ - коциклы, $[a],[b],[c]$ - соответствующие классы когомологий, причем $[a][b]=[b][c]=0$. Тогда говорят, что класс когомологий $[g]$ принадлежит тройному произведению Масси $\langle[a],[b],[c]\rangle$, если найдутся такие элементы $e, f \in C^{*}$, что $e=\bar{a} b, f=\bar{b} c$ и $g=-\bar{a} f-\bar{e} c$.

В общем случае произведение Масси - многозначная операция, которая считается тривиальной, если она содержит 0.

Далее везде мы будем полагать, что $a, b, c \in C^{*}\left(\mathscr{Z}_{K}\right)-\mathbb{Z}^{V}$-однородные клеточные коциклы. Покажем, что если ни один из классов вида $[-\bar{a} f-\bar{e} c]$, где $e$ и $f \mathbb{Z}^{V}$-однородны, не равен 0 , то произведение $\langle a, b, c\rangle$ не тривиально. Действительно, пусть $[-\bar{a} f-\bar{e} c]=0$ для некоторых $e$ и $f$. Обозначим через $e_{1}$ и $f_{1}$ их проекции на подпространства однородных элементов с теми же $\mathbb{Z}^{V}$-градуировками, что и $\bar{a} b$ и $\bar{b} c$ соответственно. Так как дифференциал сохраняет $\mathbb{Z}^{V}$-градуировку, 
легко видеть, что $d e_{1}=d e=\bar{a} b, d f_{1}=d f=\bar{b} c$. Более того, коцикл $-\bar{a} f_{1}-\overline{e_{1}} c$ является проекцией коцикла $-\bar{a} f-\bar{e} c$ на подпространство однородных элементов с теми же $\mathbb{Z}^{V}$-градуировками, что и $a b c$, и, следовательно, как и $-\bar{a} f-\bar{e} c$, гомологичен нулю. Таким образом, если в качестве новых, уже $\mathbb{Z}^{V}$-однородных, коцепей $e$ и $f$ взять $e_{1}$ и $f_{1}$, то, по-прежнему, класс $[-\bar{a} f-\bar{e} c]$ будет равняться 0 .

Используя это наблюдение, дадим метод, которьй позволяет решать вопрос о нетривиальности тройного произведения Масси в когомологиях момент-угол комплексов, не переходя к коцепям.

В дальнейшем мы везде будем пользоваться приведенным выше комбинаторным описанием кольца когомологий $\mathscr{Z}_{K}$. Рассмотрим коциклы $a \in \widetilde{C}^{*}\left(K_{\alpha}\right), b \in \widetilde{C}^{*}\left(K_{\beta}\right), c \in \widetilde{C}^{*}\left(K_{\gamma}\right)$ и соответствующие им классы когомологий $[a],[b]$ и $[c]$. Если подмножества вершин $\alpha, \beta, \gamma$ попарно пересекаются, то произведение $\langle[a],[b],[c]\rangle$ тривиально по соображениям $\mathbb{Z}^{V}$-градуировки, поэтому положим обратное. Поднимем равенство $d e=\bar{a} b \in \widetilde{C}^{*}\left(K_{\alpha \sqcup \beta}\right)$ до равенства в коцепях джойна $K_{\alpha} * K_{\beta} d\left(i_{\alpha, \beta}^{*}\right)^{-1}(e)=\bar{a} * b-\widehat{e}$. Таким образом, класс $[\widehat{e}] \in H^{*}\left(K_{\alpha} * K_{\beta}, K_{\alpha \sqcup \beta}\right)$ является прообразом класса $[a] *[b] \in \widetilde{H}^{*}\left(K_{\alpha} * K_{\beta}\right)$. Пусть $x_{1} \in C^{*}\left(K_{\alpha} * K_{\beta} * K_{\gamma}, K_{\alpha \sqcup \beta \sqcup \gamma}\right)$ - образ цикла $\overline{\widehat{e}} * c \in C^{*}\left(K_{\alpha} * K_{\beta} * K_{\gamma}, K_{\alpha \sqcup \beta} * K_{\gamma}\right)$. Для циклов $b$ и $c$ аналогично строятся $\widehat{f}$ и $x_{2}$. Теперь нетрудно видеть, что если $\delta$ - связующий гомоморфизм в точной последовательности пары $\left(K_{\alpha} * K_{\beta} * K \gamma, K_{\alpha \sqcup \beta \sqcup \gamma}\right)$, то $\delta([-\bar{a} f-\bar{e} c])=\left[x_{2}\right]-\left[x_{1}\right]$. Действительно, цикл $-\bar{a} f-\bar{e} c \in \widetilde{C}^{*}\left(K_{\alpha \sqcup \beta \sqcup \gamma}\right)$ поднимается до коцепи $-\bar{a} *\left(i_{\beta, \gamma}^{*}\right)^{-1}(f)-\left(i_{\alpha, \beta}^{*}\right)^{-1}(\bar{e}) * c \in \widetilde{C}^{*}\left(K_{\alpha} *\right.$ $\left.K_{\beta} * K_{\gamma}\right)$, теперь подсчитаем еекограницу: $d\left(-\bar{a} *\left(i_{\beta, \gamma}^{*}\right)^{-1}(f)-\left(i_{\alpha, \beta}^{*}\right)^{-1}(\bar{e}) * c\right)=-a *(\bar{b} * c-\widehat{f})+$ $(a * \bar{b}-\overline{\widehat{e}}) * c=x_{2}-x_{1}$, что и доказьвает приведенное выше равенство. Таким образом, если 0 не является элементом вида $\left[x_{2}\right]-\left[x_{1}\right]$, то тройное произведение Масси не тривиально.

Для построения примера нетривиалнного тройного произведения Масси в когомологиях момент-угол комплекса нам понадобится звездное преобразование. Пусть $\sigma \in K$ - симплекс, $\operatorname{star} \sigma=\{\tau \in K \mid \sigma \cup \tau \in K\}$ - его звезда, тогда результатом звездного преобразования $K$ по симплексу $\sigma$ является комплекс $K^{\prime}=(K \backslash \operatorname{star} \sigma) \cup \operatorname{con}(\partial \operatorname{star} \sigma)$.

Теперь мы можем получить основной резултат настоящей работы.

ТеОрема. Для любых симпличиальных сфер $K_{1}, K_{2}, K_{3}$ существует звездная перестройка $\widehat{K}$ симпличиальной сферы $K=K_{1} * K_{2} * K_{3}$ такая, что в когомология момент-угол комплекса $\mathscr{Z}_{\widehat{K}}$ существует нетривиальное тройное произведение Мас$c u$.

Ключевые шаги доказательства следуюшие. Пусть $\sigma_{1} \in K_{1}, \sigma_{2}^{\prime}, \sigma_{2}^{\prime \prime} \in K_{2}$ и $\sigma_{3} \in K_{3}-$ некоторые симплексы, причем внутренности звезд $\sigma_{2}^{\prime}$ и $\sigma_{2}^{\prime \prime}$ не пересекаются. Возьмем в качестве $\widehat{K}$ - звездное преобразование джойна $K$ по симплексам $\sigma_{1} * \sigma_{2}^{\prime} * \varnothing$ и $\varnothing * \sigma_{2}^{\prime \prime} * \sigma_{3}$. Пусть $[a],[b]$ и $[c]$ - классы из $H^{*}\left(\mathscr{Z}_{\widehat{K}}\right)$, соответствующие образующим старших размерностей сфер $K_{1}, K_{2}$ и $K_{3}$ соответственно, рассматриваемых как полные подкомплексы $\widehat{K}$.

Тогда $\langle[a],[b],[c]\rangle$ определенно и нетривиально. В самом деле, класс $[\widehat{e}] \in H^{*}\left(K_{1} * K_{2}, K_{1} *\right.$ $\left.K_{2} \backslash \operatorname{star}_{K_{1} * K_{2}} \sigma_{1} * \sigma_{2}^{\prime}\right)$ выбирается однозначно и представляет собой образующую старшей размерности этой группы когомологий, то же для класса $[\widehat{f}]$, теперь остается заметить, что так как внутренности $\operatorname{star} \sigma_{1} * \sigma_{2}^{\prime} * \varnothing$ и star $\varnothing * \sigma_{2}^{\prime \prime} * \sigma_{3}$ не пересекаются, то однозначно определенньй класс $\left[x_{2}\right]-\left[x_{1}\right]$ не равен 0 .

Автор выражает благодарность В. М. Бухштаберу, а также Т. Е. Панову за постановку задачи и неоценимую помощь и внимание, проявленное на всех этапах написания данной статьи.

\section{СПИСОК ЛИТЕРАТУРЫ}

[1] И. В. Баскаков // УМН. 2002. Т. 57. №5. С. 147-148. [2] В. М. Бухштабер, Т. Е. Панов // УМН. 2000. Т. 55. № 5. С. 3-106. 